\title{
EchoGéo
}

43 | 2018

Processus de gouvernance dans les territoires ruraux

\section{Amour des modèles, humour des modélisateurs : un dictionnaire passionnel de la modélisation urbaine et de ses usages}

Hadrien Commenges and Serge Weber

\section{(2) OpenEdition}

Journals

Electronic version

URL: https://journals.openedition.org/echogeo/15292

DOI: 10.4000/echogeo.15292

ISSN: 1963-1197

Publisher

Pôle de recherche pour l'organisation et la diffusion de l'information géographique (CNRS UMR 8586)

Electronic reference

Hadrien Commenges and Serge Weber, "Amour des modèles, humour des modélisateurs : un dictionnaire passionnel de la modélisation urbaine et de ses usages", EchoGéo [Online], 43 | 2018, Online since 22 March 2018, connection on 23 August 2021. URL: http://journals.openedition.org/ echogeo/15292 ; DOI: https://doi.org/10.4000/echogeo.15292

This text was automatically generated on 23 August 2021.

EchoGéo est mis à disposition selon les termes de la licence Creative Commons Attribution - Pas d'Utilisation Commerciale - Pas de Modification 4.0 International (CC BY-NC-ND) 


\title{
Amour des modèles, humour des modélisateurs : un dictionnaire passionnel de la modélisation urbaine et de ses usages
}

\author{
Hadrien Commenges and Serge Weber
}

1 Entretien avec Hadrien Commenges, réalisé par Serge Weber à Paris le 7 mars 2018, à propos de l'ouvrage : Bonhomme Céline, Commenges Hadrien, Deroubaix José-Frédéric (dir.), Dictionnaire passionnel de la modélisation urbaine, Paris, l'œil d'or, 2017.

2 - Serge Weber (SW). Il n'est pas courant de voir associés les deux termes de «modélisation » et de « passionnel » dans un même titre. Derrière le titre, le lecteur est agréablement surpris de voir un dictionnaire qui n'a rien d'un glossaire technique, mais au contraire des entrées, en petit nombre et assez développées, sur des interrogations de fond, qui permettent de prendre du recul sur les usages de la modélisation et sur les questions que pose cette pratique. Pourriez-vous revenir sur les intentions qui ont présidé à l'idée de cet ouvrage, et préciser dans quel esprit a été fabriqué cet ouvrage très original ${ }^{1}$ ?

3 - Hadrien Commenges (HC). L'idée de l'ouvrage est née au sein du Groupe Transversal Modélisation et dynamiques urbaines du Labex Futurs urbains, quand j'étais accueilli comme postdoctorant au LEESU (Laboratoire Eau, environnement et systèmes urbains). Ce groupe a depuis disparu mais l'esprit perdure au sein du groupe Villes et numérique (animé par José-Frédéric Deroubaix et Anne Aguilera). À l'époque, ce groupe réunissait des modélisateurs dans des domaines variés: les transports, avec des chercheurs du LVMT (Laboratoire Villes mobilités transports), la qualité de l'eau, l'assainissement (LEESU) et la qualité de l'air avec le CEREA (Centre d'enseignement et de recherche en environnement atmosphérique). Il y avait aussi des chercheurs réfléchissant aux enjeux de la modélisation en sociologie, en analyse des politiques publiques et qui, sans faire de la modélisation directement, travaillaient avec des modélisateurs. 
4 Au début, la question qui nous rassemblait était que des représentants de disciplines différentes modélisaient différemment alors même qu'ils croyaient partager un vocabulaire commun, une expérience commune, certes parfois proches mais qui n'étaient pas exactement les mêmes: nous faisions le constat que nous avions des pratiques proches et qu'il était opportun de mettre en commun nos expériences. Cela explique pourquoi le dictionnaire est devenu tout à fait autre chose qu'un glossaire technique.

5 L'idée de proposer un dictionnaire passionnel, qui ne soit pas un dictionnaire normatif, ni un conservatoire qui fixerait une norme pour toujours n'est pas originale. Mais pour autant, ce n'est pas si courant de proposer un recueil d'expériences pour montrer les dessous, ou « l'arrière-cuisine » des modélisateurs : certes, les modèles qui sont utilisés proposent de belles «sorties », bien habillées avec des belles cartes, mais en cuisine, il se passe des choses qui ne sont pas toujours aussi propres!

6 - SW. J'imagine qu'il peut s'agir par exemple des données à partir desquelles les modèles sont construits?

7 - HC. Oui, mais pas seulement. Par exemple, un certain nombre de critiques ont été formulées à l'encontre des modèles dits intégrés, qui comportent plusieurs modules, un module qui est un modèle de choix résidentiel, puis un module modélisant les mobilités quotidiennes, et le modèle intégré opère un couplage entre les deux, en l'occurrence des choix résidentiels corrélés avec des profils sociaux et leurs mobilités quotidiennes. Chaque modèle pouvant être constitué de plusieurs modules couplés les uns aux autres. Le problème de ces modèles intégrés c'est que, même avec seulement deux modules, il est très difficile d'évaluer la qualité des sorties du modèle. Quand chaque module contient une erreur, même minime, il y a une propagation de l'erreur, et le modèle devient impossible à évaluer : il est possible qu'une petite erreur dans l'un entraîne une énorme erreur dans l'autre. Un modèle couplant un grand nombre de modules, on dira que c'est une "usine à gaz», est inévaluable. Il est toujours possible d'habiller les sorties avec un discours raisonnable, mais personne ne peut dire ce qui joue dans le modèle.

8 L'ambition du dictionnaire, c'était de montrer la pratique de la modélisation, pas seulement ses résultats ni sa définition, mais le travail et les pratiques des personnes qui construisent et qui utilisent les modèles. Et ce projet avait un objectif de divulgation, de vulgarisation, étant donné que beaucoup de gens se retrouvent, souvent sans le savoir, bénéficiaires ou victimes des modèles. Partout, (par exemple le schéma d'assainissement de l'île-de-France, le Grand Paris Express), on peut trouver des modèles qui ont un impact sur la vie des gens. Nous voulions donc toucher un public autre que la communauté professionnelle des modélisateurs.

9 - SW. Y avait-il d'autres ambitions dans ce projet de dictionnaire ?

10 - HC. Nous avions aussi envie de "nous faire plaisir », en écrivant autre chose que les articles habituels, systématiquement assortis d'un lourd appareil bibliographique qui peut rebuter les lecteurs en dehors du monde académique, et d'écrire, sans devoir référencer chacune des affirmations, un texte qui soit facile et agréable à lire.

11 - SW. En effet, il y a beaucoup de passages humoristiques, maniant avec habileté le second degré, c'est ce qui rend l'ouvrage particulièrement savoureux.

12 - HC. Nous avons été plus ou moins à l'aise avec ce style, car aucun de nous n'avait jamais fait l'expérience d'écrire en dehors des canons d'écriture scientifique. Certains 
textes sont plus humoristiques que d'autres. Dans certains textes qui ne sont pas ouvertement drôles, on voit que les auteurs ont une verve, une plume; d'autres auteurs ont découvert que ce n'était pas si facile d'écrire autrement qu'avec les canons de l'écriture scientifique, mais ils y ont pris goût.

13 Dans les critères initiaux de l'écriture, il fallait qu'il y ait à la fois un contenu scientifique identifiable et un style de divulgation, un style non académique, si possible humoristique. On peut mentionner par exemple la comparaison entre le modélisateur qui se retrouve à présenter son (mauvais) modèle pour la première fois et l'ami, peintre amateur sans talent, qui nous met face à son «encombrante toile (entrée «Validation », Pierre-Antoine Versini).

Ou encore, l'entrée "Scénario" (Isabelle Coll): "si vous ne déconnez pas avec le scénario, habituellement tout se passe bien ». Cette entrée moque la prolifération des sigles désignant les modèles et les projets et propose une réflexion sur leur côté "bankable ». Ce qui touche à un aspect très important: il y a un aspect clairement " capitaliste » lorsqu'on conçoit un modèle. On met du temps à le mettre en place, mais une fois qu'il existe, il peut devenir rentable, car on peut produire de nouveaux résultats à peu de frais. C'est un lourd investissement initial, mais, du point de vue de la publication scientifique, le coût marginal d'une nouvelle publication est très faible. Certains modèles ont été très coûteux, ont été développés sur une très longue durée, et ont été suivi d'un très grand nombre de publications. Il y a donc une rentabilité qui explique le succès de la modélisation, sa rentabilité scientifique (publications) et aussi sa rentabilité économique (expertise).

15 - SW. Pourriez-vous proposer quelques thèmes clefs, des notions importantes, abordées dans les différentes entrées du dictionnaire, dont les articles permettent la divulgation?

16 - HC. Je serai plus à l'aise si je me limite à répondre sur mes propres thèmes, qui se situent essentiellement dans le domaine STS (sciences, technologies et sociétés), notamment l'usage de la quantification pour l'aménagement, dans la mise en place de politiques publiques.

17 - SW. Faites-vous référence à l'ouvrage de Lascoumes et Le Galès (2005), Gouverner par les instruments, que vous citez dans vos écrits?

18 - HC. Un de mes thèmes de prédilection, ces sont les instruments de quantification, qui sont faits pour décrire mais qui deviennent performatifs. Prenons par exemple le mot "indicateur». Quand on commence à définir le terme "indicateur", on parle de l'index, le «doigt qui montre». Dans l'entrée «indicateur» je file la petite phrase chinoise : " quand le doigt montre la lune, l'imbécile regarde le doigt ». Alors qu'en fait c'est bien le doigt qui est important. On devrait donc dire : « quand le doigt montre la lune, l'imbécile regarde la lune ». Il y a une série de questions intéressantes à poser concernant le doigt qui montre la lune : comment la montre-t-il, de manière autoritaire ou pédagogique? Pourquoi désigne-t-il la lune et pas autre chose ? Son geste revient-il à masquer autre chose? Est-ce un préambule à une autre action? À qui appartient ce doigt?

19 - SW. Donc, d'une certaine manière, cela reviendrait à se demander si c'est un « savoir situé ", comme cela a été proposé dans d'autres champs des sciences humaines et sociales? 
20 - HC. Oui. Et c'est aussi une entrée vers la prescription. Le doigt montre la lune pour certaines raisons, et on peut supposer que ce n'est pas une simple action descriptive parce que les indicateurs qui sont mis en place pour décrire finissent par agir. On peut en trouver une illustration dans le principe de description autour duquel s'articule la série The Wire. C'est justement le fil directeur de la série : chaque saison se passe dans un monde social donné, avec à chaque fois des indicateurs descriptifs qui deviennent performatifs, que ce soit dans le monde de la police, du système scolaire, du monde des médias, etc.

21 - SW. Cette démarche pourrait-elle s'apparenter à une approche critique de la modélisation?

22 - HC. Oui, le dictionnaire est critique. Au début nous souhaitions l'appeler « dictionnaire amoureux » mais nous avons découvert que l'expression est une marque déposée par Plon, c'est pourquoi nous l'avons appelé "passionnel ». Ce terme lui va bien, car il contient à la fois le conflit et l'attachement de la relation amoureuse. Le propos est critique parce que nous nous envoyons des piques à nous-mêmes, mais nous l'aimons bien, notre domaine.

23 - SW. Peut-on dire qu'il est essentiellement critique des usages qui sont faits des modèles?

24 - HC. En partie oui, dans la mesure où il peut se trouver de mauvais usages des modèles. L'exemple typique est la modélisation qui a précédé la mise en place de l'Orlyval, au début des années 1990. C'était à une époque très marquée par la pensée Thatcher avec l'idée que les transports publics pouvaient ou devaient être rentables. C'est une aberration, car les transports publics coûtent nécessairement de l'argent public. S'ils étaient rentables, ils ne seraient justement pas publics.

25 - SW. Cette idée qui a circulé à cette époque appartient-elle au passé ?

26 - HC. Oui, c'est du passé. Les modèles ont été faits et sans doute «torturés " pour leur faire dire que l'Orlyval allait être rentable et cela a été un fiasco, les financeurs ont abandonné le projet au bout d'un an ou deux. Au début c'était un consortium, avec le Crédit Lyonnais, la Caisse des Dépôts et d'autres acteurs financiers. Après la banqueroute, la RATP et la Région reprennent cette affaire "rentable». Aujourd'hui encore les modalités de financement de l'Orlyval sont particulières, et c'est pour cela qu'il n'est toujours pas intégré au Pass Navigo.

27 - SW. Revenons-en à cette question : qu'est-ce que le dictionnaire a de critique ?

28 - HC. Dans une certaine mesure, il est critique vis-à-vis des mauvais usages des modèles. Mais il est aussi critique vis-à-vis d'une attitude qui relèverait de la naïveté technologique, qui voudrait que les résultats des modèles soient directement injectés dans la mise en place des politiques publiques. C'est une idée naïve de croire qu'il y a un décideur unique et que "le » décideur va prendre «la » décision. En fait il y a tout un champ de recherche qui établit une critique de la décision. Pierre Muller (2000) dit notamment qu'il n'y a pas de décision distincte et observable seulement une "décantation progressive des choix». Cette critique de la décision existe depuis longtemps en science politique. Tout ce qu'on peut dire c'est que les instruments quantitatifs, comme les modèles, interviennent ou influencent, vaguement, indirectement, à un terme moyen, les personnes qui sont impliquées dans la " décantation progressive des choix ». Ils peuvent notamment servir à éclairer le cadre cognitif des acteurs qui interviennent dans cette décantation. 

nécessaire, c'est la séduction $»$. On pourrait appliquer cette phrase aux modèles. La qualité des estimations des modèles, c'est le luxe ; le nécessaire, c'est qu'il soit « sexy » en apparence. C'était l'occasion de filer la métaphore à propos de ce qu'est un modèle « sexy ». Quand un comité technique utilise un modèle pour une décision, personne n'a vu le modèle en tant que tel, on ne voit que des sorties de modèles, des graphiques, des cartes. Si les sorties sont sexy, la séduction du modèle opère, quelle que soit la qualité du modèle en lui-même.

33 Le dessin qui illustre cet article est particulièrement réussi dans le registre du second degré, il représente un hexagone (pris comme modèle de la France) en sous-vêtements. Il invite à prendre conscience que derrière toute démarche en modélisation, comme entrée, nous avons le réel. Le modèle simplifie énormément le réel pour pouvoir le manipuler et produire une sortie qui est elle-même ré-habillée pour ressembler au réel. La sortie peut être présentée comme un «retour au réel ». C'est là que la séduction opère, lorsque le modèle recrée quelque chose qui ressemble au réel initial.

34 - SW. Les illustrations réussissent en effet toujours à interpeler le lecteur, ce qui n'était pas un pari gagné d'avance! Comment avez-vous travaillé avec l'illustratrice Elsa Hieramente?

35 - HC. Les illustrations sont le fruit d'un mélange des idées propres de la dessinatrice et des idées des membres du groupe, mis en forme par elle. Il y a eu tout un va-et-vient entre quelques auteurs qui exposaient une idée et la dessinatrice qui leur donnait forme, comme une traduction sous forme d'image. Voilà par exemple le dessin présentant la question de l'optimisation de l'espace dans les transports: «homme debout, homme assis, homme optimisé (représenté plié en quatre)». La dessinatrice, sans être familière des modèles, faisait une proposition dessinée à partir d'un texte ou d'une idée proposée par l'auteur.

36 Cet autre dessin par exemple joue sur l'homophonie entre "dis simulation" et «dissimulation ». Il n'y a pas toujours «dissimulation» dans la simulation, mais ça renvoie à l'idée de séduction dont on parlait avant, si on arrive à bien habiller la sortie $\mathrm{du}$ modèle, personne ne critiquera sa validité, du moins pas dans le monde opérationnel de l'aménagement. Dans le champ de la modélisation, il y a toute une réflexion autour de l'erreur. Les modélisateurs sont formés à détecter, à évaluer 
l'erreur. Mais face à un élu, à un comité technique, à un opérationnel, le modélisateur n'est jamais récompensé pour parler de l'erreur, mais beaucoup plus pour la camoufler. C'est le côté schizophrène de son travail : au moment de présenter son modèle il parlera le moins possible de l'erreur attachée au modèle alors que c'est son obsession au moment de concevoir le modèle.

- SW. Est-ce que cela se vérifie indifféremment du fait que le commanditaire soit public ou privé ?

38 - HC. De ce que je sais, dans les exemples que je connais, le commanditaire est toujours public, et le modélisateur est soit privé, soit public. Quand je dis public, ça signifie financé avec de l'argent public, même si le statut juridique est privé. Mais ce n'est pas vrai dans tous les secteurs. Par exemple, dans les transports, les grands modèles sont développés par des organismes publics comme la DRIEA (Direction régionale et interdépartementale de l'équipement et de l'aménagement d'île-de-France - ancienne DREIF) ou comme le STIF (Syndicat des transports d'Île-de-France, devenu Île-de-France mobilités), qui a son propre modèle. Mais pour faire le schéma directeur d'assainissement de Paris et de la petite couronne, là il y a un bureau d'études en ingénierie hydraulique qui intervient pour le SIAAP (Syndicat interdépartemental d'assainissement de l'agglomération parisienne) : le commanditaire est public et, dans le processus, il y a un bureau d'études privé qui vend son expertise, alimentée par un modèle.

39 - SW. Si on résume, on peut dire que c'est la dimension "science appliquée " de la modélisation qui fait le cœur de l'ouvrage.

40 - HC. Oui, c'est ça. Dans le domaine de l'aménagement, des transports, de l'assainissement, de la qualité de l'air, il y a de nombreuses applications des modèles. D'ailleurs ces domaines sont peuplés d'étudiants formés au sein des laboratoires de notre groupe et parfois par les auteurs du dictionnaire.

41 - SW. Cela témoigne d'un changement dans la manière d'aborder les modèles en géographie...

42 - HC. Oui et non. En ce qui concerne la géographie, il faut voir qu'il y a une différence entre la modélisation à visée théorique et la modélisation appliquée. La géographie théorique qui apparaît dans les années 1960 se pose des questions sur la possibilité d'existence de lois dans le monde social, alors que les sciences de l'ingénieur ne se posent pas du tout cette question. Elles se posent une question appliquée : « connaître une régularité peut-il avoir une application concrète ?».

43 - SW. Pour autant, ce n'est pas un dictionnaire de sciences de l'ingénieur.

44 - HC. Non, en effet. Disons que c'est un dictionnaire sur l'usage des modèles pour l'aménagement. Il existe toute une littérature en science politique, qui étudie les processus décisionnels et la manière dont les instruments quantitatifs entrent en jeu dans ce processus, donc ce n'est pas vraiment les sciences de l'ingénieur, mais plutôt la science politique. En fait, il y a un fossé entre d'un côté les modélisateurs qui viennent des sciences de l'ingénieur, qui savent développer des modèles, mais qui ne s'interrogent pas vraiment sur les usages qui en sont faits, et de l'autre côté, les personnes qui en font un usage et ne savent pas comment les modèles ont été développés. Les usages réels des modèles dans l'aménagement, c'est une niche de questionnements sur laquelle très peu de chercheurs ont écrit. 
45 - SW. Peut-on dire que ce lien entre usage des modèles et conception des modèles reste à faire?

46 - HC. Oui en partie. Dans la lignée des sociologues comme Latour (1996 [1979]) qui sont allés voir dans un laboratoire comment les scientifiques font de la science, la science « en train de se faire ». Presque personne n'est allé observer comment, dans un comité technique, les modèles sont utilisés au sein du comité, pour voir le modèle «en train d'être utilisé ». C'était l'objet de mon post-doctorat.

47 - SW. Cette question a donc trouvé un écho dans l'équipe du dictionnaire?

48 - HC. Oui bien sûr. C'était une question importante, pour José-Frédéric Deroubaix et Céline Bonhomme, dans la mesure où ce sont eux qui avaient proposé le sujet de postdoctorat. Et aussi pour Olivier Bonin, Fabien Leurent et Christian Seigneur qui formaient le comité d'encadrement. C'est une question qu'avait commencé à creuser Lorenza Tomasoni qui occupait le poste avant moi et qui a donné lieu à un article ("Who is the expert»? 2017) et un chapitre d'ouvrage.

49 Mais c'est un thème très rare, sur lequel il $\mathrm{y}$ a peu de publications. Il $\mathrm{y}$ a eu nombre d'articles dans les années 1970, dans la lignée du célèbre «Requiem for large scale models ", (Lee, 1973), à propos de ces modèles intégrés. Vingt ans plus tard, des auteurs ont réagi et se sont interrogés sur le fait que dans les années 1970, ces modèles ont périclité. Ces auteurs ont donné des explications essentiellement techniques, selon lesquelles le déclin de ces modèles s'expliquait parce qu'ils étaient trop gourmands en puissance de calcul et en données. Peu de personnes sont allées voir comment ces modèles étaient utilisés dans les agences d'urbanisme et d'aménagement. Une seule personne, Gary Brewer, est allée observer les modèles en train d'être utilisés, allant à l'encontre d' "un monopole de l'explication technique »: " to the extent that the model could answer questions, they were questions that nobody was asking" (Brewer, 1973). En somme, comme la modélisation se situe dans un domaine technique, avec des compétences techniques, pour les techniciens, tout se résume à une question technique : pour eux, le succès ou l'échec d'un modèle est dû à la bonne ou à la mauvaise qualité de la technique, alors qu'il y a toute une série de contre-exemples qui montrent que cela peut être lié à autre chose que de la technique. Un exemple dans un autre domaine, celui des cassettes vidéo : la rivalité entre cassettes VHS et Betamax a mené à l'échec du standard Betamax. Techniquement ces cassettes étaient aussi bonnes que les VHS, les explications du succès de l'une et de l'échec de l'autre sont organisationnelles, liées aux positions des entreprises sur le marché à cette époque. De la même manière, il faut expliquer le déclin de ces gros modèles par des facteurs organisationnels, ils ne remplissaient aucun besoin. S'il est peu habituel de poser la question de cette manière, c'est sans doute parce qu'un domaine technique est peuplé de techniciens qui voient avant tout le côté technique.

50 - SW. En d'autres termes, on pourrait conclure que le Dictionnaire passionnel est essentiellement un dictionnaire de vigilance sur l'expertise?

51 - HC. Oui, c'est tout à fait cela. Le dictionnaire contient une entrée sur l'expertise (par José-Frédéric Deroubaix), qui rappelle le succès d'expressions telles que « faire parler le modèle ", "que dit le modèle ", etc., comme si le modèle pouvait parler. Cela ouvre le débat sur la démocratie technique. Il écrit: «à mesure que l'outil expert remplace l'individu expert, plus personne n'est réellement responsable d'une erreur d'évaluation ». C'est une question de dépolitisation, et c'est un phénomène plus global, 
on analyse les politiques publiques, on va transférer des décisions politiques dans des champs techniques à un comité technique, qui aura des outils techniques.

En conclusion je voudrais quand même insister sur l'objectif de ce livre. D'une certaine manière, ce dictionnaire suppose qu'un modélisateur aurait le droit de se moquer des modélisateurs, mais nous ne voulons surtout pas décrédibiliser cette pratique, dire qu'elle est inutile ou qu'elle n'a pas de valeur scientifique. C'est une pratique qui a ses limites, comme toutes les pratiques scientifiques. Je crois que tous les auteurs du livre partagent l'idée qu'un modèle a sa place dans un processus de décision. Un des constats qui nous avait interpelés, lorsque des gens disent que plus les problèmes sont complexes plus ils ont besoin d'instruments sophistiqués pour les résoudre, c'est qu'au contraire, plus les configurations décisionnelles et systèmes d'acteurs sont complexes, plus il faut des outils pour faire du lien, du lien social, du langage commun entre tous les acteurs qui prennent des décisions, et c'est précisément un des avantages du modèle. Le modèle permet de réfléchir collectivement: il ne donne pas la bonne solution à un problème identifié mais sert de support à une réflexion collective.

\section{BIBLIOGRAPHY}

Brewer G. D., 1973. Politicians, Bureaucrats, and the Consultant: a Critique of Urban Problem Solving. New York, Basic Books.

Commenges H., Tomasoni L., Seigneur C., Bonin O., Leurent F., Bonhomme C., Deroubaix J.-F., 2017. Who is the Expert? Integrated Urban Modeling and the Reconfiguration of Expertise. Journal of Urban Technology, vol. 24, nº 2, p. 89-108.

Commenges H., Tomasoni L., Seigneur Ch., Bonin O., Leurent F., Bonhomme C., Deroubaix J.-F., 2016. L'expertise est-elle soluble dans la modélisation intégrée? In Némery J.-C. et Thuriot F. (dir.), Les instruments de l'action publique et les dispositifs territoriaux, L'Harmattan, p. 59-74.

Goodman N., 1976 [1 1 ère édition 1968]. Languages of Art. An Approach to a Theory of Symbols, Indianapolis, Hackett.

Lascousmes P., Le Galès P. (dir.), 2005. Gouverner par les instruments, Paris, Presses de Sciences-Po. Latour B., Woolgar S., 1996 [1979]. La vie de laboratoire. La production des faits scientifiques. Paris, La Découverte.

Lee Douglass B. Jr., 1973. Requiem for Large-Scale Models. Journal of the American Institute of Planners, vol. 39, $\mathrm{n}^{\circ}$ 3, p. 163-178.

Muller P., 2000. L'analyse cognitive des politiques publiques : vers une sociologie politique de l'action publique. Revue française de science politique, vol. 50, $\mathrm{n}^{\circ} 2$, p. 189-208. 


\section{NOTES}

1. Les auteurs qui ont participé à cet ouvrage sont Céline Bonhomme, José-Frédéric Deroubaix, Olivier Bonin, Isabelle Coll, Hadrien Commenges, Amandine de Coninck, Bernard de Gouvello, Natalia Kotelnikova-Weiler, Joël Idt, Florent Le Néchet, Martin Seidl, Christian Seigneur, Mathilde Soyer, Claire Trinh, Pierre-Antoine Versini. Les illustrations ont été réalisées par Elsa Hieramente.

\section{AUTHORS}

\section{HADRIEN COMMENGES}

Hadrien Commenges, hadrien.commenges@univ-paris1.fr, est Maître de conférences à l'Université Paris 1, membre de l'UMR Géographie-Cités et Chercheur associé au LEESU - École des Ponts ParisTech.

\section{SERGE WEBER}

Serge Weber, serge.weber@u-pem.fr, est Professeur d'université en géographie à l'UPEM (Université Paris-Est Marne-la-Vallée) et membre du laboratoire ACP. 